Original Research Paper

\title{
The Role of Perspective-Taking on Ability to Recognize Fear
}

\author{
${ }^{1}$ Andrea Trubanova, ${ }^{2}$ Inyoung Kim, ${ }^{1}$ Marika C. Coffman, ${ }^{1}$ Martha Ann Bell, \\ ${ }^{1}$ J. Anthony Richey, ${ }^{3}$ Stephen M. LaConte, ${ }^{4}$ Denis Gracanin and ${ }^{1}$ Susan W. White \\ ${ }^{I}$ Department of Psychology, Virginia Polytechnic Institute and State University, Blacksburg, Virginia, USA \\ ${ }^{2}$ Department of Statistics, Virginia Polytechnic Institute and State University, Blacksburg, Virginia, USA \\ ${ }^{3}$ Department of Biomedical Engineering and Sciences, Virginia Tech Carilion Research Institute, Roanoke, Virginia, USA \\ ${ }^{4}$ Department of Computer Science, Virginia Polytechnic Institute and State University, Blacksburg, Virginia, USA
}

Article history

Received: 23-09-2015

Revised: $10-11-2015$

Accepted: 28-12-2015

Corresponding Author:

Andrea Trubanova,

Department of Psychology,

Virginia Polytechnic Institute and

State University, Blacksburg,

Virginia, USA

Tel: (540)231-2024

Fax: (540) 231-8193

Email: atruban@vt.edu

\begin{abstract}
Impairment in the ability to detect certain emotions, such as fear, is linked to multiple disorders and follows a pattern of interindividual variability and intra-individual stability over time. Deficits in fear recognition are often related to social and interpersonal difficulties but the mechanisms by which this processing deficit might occur are not well understood. One potential mechanism through which impaired fear detection may influence social competency is through diminished perspective-taking, the ability to perceive and consider the point of view of another individual. In the current study, we hypothesized that intraindividual variability in the accuracy of facial emotion recognition is linked to perspective-taking abilities in a well-characterized, non-clinical adult sample. Results indicated that the ability to accurately detect fear in the faces of others was positively correlated with perspective-taking, consistent with initial hypotheses. This relationship appeared to be unique to recognition of fear, as perspective-taking was not significantly associated with recognition of the other basic emotions. Results from this study represent an initial step towards establishing a potential mechanism between some processes of FER and perspective-taking difficulties. It is important to establish the relationship between these processes in a non-clinical adult sample so that we can consider the possibility of a developmental or pathological influence of impoverished perspective-taking on fear perception.
\end{abstract}

Keywords: Perspective-Taking, Emotion Recognition, Fear, Empathy

\section{Introduction}

Facial emotion recognition, the ability to accurately identify and interpret facial expressions emerges early in life and is crucial for effective social interaction. Impaired facial emotion recognition has been observed in neurological and psychiatric disorders (Donno et al., 2010). Specifically, impaired ability to detect another person's fear, a distressing emotion aroused by threat of impending or possible danger, has been linked to many types of psychopathology (Gross and Jazaieri, 2014). However, the association between ability to recognize facial expressions and social functioning has not been well studied in healthy, non-psychiatric sample. Outside of psychopathology, it is important to see if and how fear recognition differs from recognition of other emotions in non-clinical individuals. Although the mechanisms through which impoverished fear detection might cause interpersonal difficulties are not well understood, one potential process is impaired perspective-taking, an ability to consider the world from other viewpoints and "allows an individual to anticipate the behavior and reactions of others" (Davis, 1983, p. 115). Accuracy of facial expression recognition may be a primary determinant of observed individual differences in processes such as interpersonal insight and perspective-taking. Thus, the goal of the current study was to examine the association between perspective-taking and emotion recognition in a well characterized, non-clinical adult sample.

The development of emotion recognition appears to be important for normative interpersonal functioning, as 
the ability to recognize and label emotion expressions predicts positive social interactions, as well as academic competence in young children (Izard et al., 2001). Conversely, difficulties with emotion recognition have been implicated in many forms of psychopathology (Gross and Jazaieri, 2014) including early-onset conduct disorder (Fairchild et al., 2009), depression (Dalili et al., 2014), Autism Spectrum Disorder (ASD; Uljarevic and Hamilton, 2013) and psychopathic traits (Dadds et al., 2008). Although it is possible that an individual can have broadly impaired recognition across a range of emotions, there is considerable evidence that impairment in recognition of specific emotions, fear in particular, is linked to psychopathology.

Collectively, extant research suggests that impaired fear recognition may be a stronger predictor of psychopathology than impairment in the recognition of other emotions (Demirel et al., 2014; Montagne et al., 2005). Impaired recognition of fear has been found to be associated with criminal behaviors among people with schizophrenia (Weiss et al., 2006) and there is a robust association between inability to perceive fear via facial cues, antisocial behavior and a lack of empathy (Blair et al., 2001; Montagne et al., 2005; Stevens et al., 2001). Impairment in the ability to perceive fear has also been documented in bipolar disorder (Demirel et al., 2014), ASD (Humphreys et al., 2007) and ADHD (Aspan et al., 2014). Collectively, impairment in fear recognition has been observed across multiple disorders that share difficulties in social interaction. In light of the social and interpersonal difficulties that may result from impaired fear recognition (Corden et al., 2008; Skuse, 2003), an improved understanding of this process may facilitate the development of new intervention concepts that are applicable across a range of psychiatric disorders characterized by social disability. One such potential mechanism is perspective-taking, a multi-faceted construct often impaired across disorders. Both perspective-taking and facial emotion recognition are necessary aspects for successful social interactions.

Perspective-taking refers to the ability to perceive, appreciate and consider the perspective, or point of view, of another individual. It has long been recognized as a critical aspect for successful social interaction. Studies in clinical and non-clinical samples have shown a positive association between perspective-taking and interpersonal skills (Gerace et al., 2013; Marsh et al., 1981), highlighting the importance of perspective-taking in everyday interactions. Many of the populations struggling with decreased perspective-taking also show deficits in emotion recognition. For example, a study by Seidel et al. (2013) found reduced accuracy in violent offenders compared to non-psychiatric controls in emotion recognition and an association between a high number of violent assaults and decreased accuracy in perspective-taking for angry scenes. In addition, deficits in perspective-taking have been implicated in a subset of individuals with conduct disorder who show low callous-unemotional traits (AnastassiouHadjicharalambous and Warden, 2008) and in individuals with ASD (Baron-Cohen et al., 2000), both of which are clinical populations characterized by social problems and who often show deficits in emotion recognition abilities.

Emotion recognition emerges early in life, with newborns being able to discriminate among some facial expressions (Field et al., 1982). The early roots of perspective-taking, on the other hand, are not present until after the first year (Sodian et al., 2007), with higher-level perspective-taking abilities emerging around 4 or 5 years of age (Frick et al., 2014). As such, it may be that weakened recognition of fear precedes impaired perspective-taking developmentally. Although these processes are conceptually and developmentally distinct, linkages between the two have been established by intervention studies showing that treatments addressing social cognition impairments, such as diminished perspective-taking, often affect emotion recognition in tandem. For example, a study by Gibson et al. (2014) showed that administration of oxytocin to adults with a schizophrenia diagnosis not only improved perspective-taking, but also improved fear recognition. Nantel-Vivier et al. (2011) found that boys with elevated physical aggression, when given tryptophan, improved in both their perspective-taking and ability to distinguish facial expression of fear. It is important to establish a relationship between these concepts outside of a treatment study. Even highly focused treatments often influence other, non-targeted processes indirectly (Borkovec et al., 1995), suggesting that observation of change in a secondary construct (e.g., emotion recognition) does not establish its relationship to the target outcome (e.g., perspectivetaking). In order to identify candidate mechanisms for prevention or intervention efforts in clinical samples, links between the proposed mechanisms and behaviors of interest must first be established. Secondly, in order to understand the relationship between processes across disorders as well as outside of psychopathology (i.e., as continuously distributed individual human differences), it is important to explore them concurrently, in a non-clinical population.

Based on prior research showing that emotion recognition temporally precedes perspective-taking developmentally and that altering perspective-taking can secondarily alter fear recognition ability, we 
propose that perspective-taking and impaired fear recognition ability are related processes. In the present study, we sought to examine the connection between recognition of fear, relative to the other basic emotions and perspective-taking. Specifically, we hypothesized that there is a positive association between perspectivetaking and accuracy for recognition of fear in nonclinical, adult sample.

\section{Method}

\section{Participants}

A sample of 20 non-clinical adult males enrolled into a larger study evaluating emotion recognition accuracy through electroencephalography (EEG) and functional Magnetic Resonance Imaging (fMRI). Participants were recruited through flyers posted in areas near the University campus (e.g., coffee shops and on-campus buildings) as well as through the Department of Psychology website and through the University Graduate School. Interested participants were directed to contact the study investigator by phone or email. Only the behavioral data from the project are analyzed and reported in this study. Table 1 displays the participant characteristics. Inclusion criteria required participants to be: (1) Male; (2) between ages 18 to 28, inclusive; (3) of at least average cognitive ability (IQ equal to or above 80 ), as confirmed by Wechsler Abbreviated Scale of Intelligence, $2^{\text {nd }}$ edition (WASI-II; Wechsler, 2011); (4) capable of undergoing imaging (no MRI contraindications such as metal in body); (5) healthy with no known genetic, medical, or neurological conditions; (6) ambulatory with no known, uncorrected sensory deficits; (7) psychologically healthy (no diagnosed mental health conditions such as depression and anxiety, as confirmed by a clinical interview (Anxiety Disorders Interview Schedule for DSM-5 [ADIS-5, Silverman and Albano, 1996]); and (8) using no psychotropic medications. Females were excluded given the nature of the broader study with the focus on neural response to social stimuli, which has been shown to differ between sexes, reflecting influential differences in social information processing between males and females (Coffman et al., 2015). In addition, females were excluded given the power needed to explore sex differences within the study. A young adult sample was utilized because the focus of the broader study was to design a facial emotion recognition intervention developed for use by adults. Eight additional participants completed the assessment portion of the study but were excluded due to moving out of state $(n=1)$, meeting criteria for mental health condition $(n=4)$, consistent and current drug use $(n=2)$, or not attending subsequent scheduled sessions $(n=1)$.

\begin{tabular}{lll}
\multicolumn{3}{l}{ Table 1. Demographics and descriptive data $(n=20)$} \\
\hline Race & $n(\%)$ & $\mathrm{M}(S D)$ \\
$\quad$ Caucasian & $9(45 \%)$ & \\
African American & $4(20 \%)$ & \\
Asian & $4(20 \%)$ & \\
$\quad$ Mixed & $3(15 \%)$ & \\
Education Level & & \\
$\quad$ High School Diploma & $0(0 \%)$ & $23.4(2.87)$ \\
$\quad$ Some College & $7(35 \%)$ & $110(8.64)$ \\
$\quad$ College Diploma & $8(40 \%)$ & $5(25 \%)$ \\
$\quad$ Graduate School & & \\
Age & & \\
IQ & &
\end{tabular}

\section{Procedure}

Interested participants completed an initial phone screen to answer preliminary eligibility questions, followed by one in-lab session to confirm study eligibility (e.g., clinical interview, cognitive assessment). If they met all inclusion criteria, participants were then scheduled for two experimental visits (the EEG and fMRI portions of the study completed for purposes of a larger study). The order of the two visits was counterbalanced so that half of the participants completed the EEG session first and the other half of the participants completed the fMRI session first. The two experimental visits were scheduled to occur within 7 to 21 days of each other. During these visits, participants completed the emotion recognition task and the remaining behavioral measures and questionnaires. The two sessions were otherwise equal in the task that participants completed, described below. All participants received \$25 for each session, for a total of $\$ 75$ for completion of all three in-person visits.

\section{Perspective-Taking}

Perspective-taking was measured with the Interpersonal Reactivity Index (IRI; Davis, 1980), a 28item self-report scale of empathic ability, such as ability to take another's perspective. Items are answered on a 5point Likert scale ranging from "does not describe me well" to "describes me very well". The total IRI score is comprised of four subscales: Perspective-taking, Fantasy, Empathic Concern and Personal Distress. Internal reliability ranges from alpha $=0.70$ to 0.78 for the four subscales, indicating that the measure appears to reliably tap four separate empathy factors (Davis, 1980). The Perspective-taking subscale measures the tendency of the participant to take the point of view of others (e.g., "When I am upset at someone, I usually try to 'put myself in his shoes' for a while"). Only the Perspective-taking subscale was examined for this study due to the specific focus on perspective-taking, a cognitive empathy, which is 
often dissociated from the emotional empathy in clinical populations (e.g., Dziobek et al., 2008).

\section{Emotion Recognition}

Emotion Recognition was assessed through a behavioral task completed during fMRI and EEG data collection, for purposes or a larger study. The images were selected from the Cohn-Kanade image database (http://www.pitt.edu/ emotion/ck-spread.htm), due to a number of factors that ensure consistency among the videos, including: direct gaze of actors, size and distance of the faces from the camera and plain backgrounds. Further, all images have been Facial Action Coded and Emotion Facial Action coded, meeting the standards for portraying prototypical emotional expressions (Kanade et al., 2000). Lucey et al. (2010) evaluated the validity of the emotion labels and found acceptable machine-based agreement between presented and detected emotion. For this study, the images from the Cohn-Kanade image set were individually reviewed and consensus rated by two graduate research assistants for authenticity of the emotion. Selected face images portrayed six basic emotions (i.e., anger, fear, disgust, surprise, happiness, sadness), such that there were 10 image sets for each emotion. The number of times an individual actor was portrayed was limited to three presentations, in order to reduce participant familiarity with the actor. Only male faces were selected, due to the all-male participant sample. The facial emotions were shown through a series of still images (which were compiled into a dynamic video, as described below). The final group of 60 videos was then split into 2 sets (for two tasks), each comprised of 30 emotion videos, so that no videos were repeated within a visit. Only one task was analyzed for this study and therefore the data includes information from 30 stimuli repeated during the two visits. Each actor was shown no more than 2 times per condition and each emotion was shown 5 times per task. The videos were created using custom MATLAB code that interpolated the space between the still images to make the videos appear smoother. On average, the videos were $20.57 \mathrm{sec}(S D=7.26)$ in length across different emotions and did not vary in length across emotional conditions. The presentation of stimuli was randomized for each participant. The participant was asked to respond with a button press as soon as he recognized the emotion. After the completion of the video, the participant was asked to select the portrayed emotion from a list of six presented emotions.

\section{Results}

There was no difference between EEG and fMRI sessions in terms of accuracy in the emotion recognition paradigm for any of the emotions [all $F(1$, $18)<1.92$, all $p>0.18$ ] or in perspective-taking scores $[F(1,18)=1.80, p=0.20]$. Data were therefore combined across the two sessions.

\section{Emotion Recognition and Perspective-Taking Scores}

Scores on the perspective-taking subscale of the IRI ranged from 10 to $26(M=19.60, S D=4.08)$. Average accuracy for recognition of emotion, collapsed across emotions, was $75.33 \%(S D=8.54)$. See Fig. 1 for the average accuracy for each of the six emotions. Table 2 portrays the misattribution matrix illustrating the emotion participants chose when they did not select the correct emotion. There was a difference in accuracy among emotions as determined by one-way ANOVA, $F(5,114)=16.898, p<0.001$. Post hoc comparisons using the Tukey HSD test indicated higher accuracy for happy $(M=99.00, S D=$ $4.47)$, sad $(M=90.00, S D=15.22)$ and surprise $(M=$ $88.00, S D=19.89)$ compared to anger $(M=55.00, S D$ $=21.40)$, disgust $(M=64.00, S D=26.44)$ and fear $(M$ $=56.00, S D=28.73)$. However, accuracy for anger, disgust and fear did not differ from each other (all $p$ 's $>0.75$ ) and accuracy for happy, sad and surprise emotions were not different from each other (all $p$ 's $>0.56)$. When incorrectly identified, emotions were not reciprocally or equally misidentified (e.g., surprise as fear and fear as surprise). Rather, as shown in Table 2, surprise was most often confused with fear, fear was most often confused with disgust and disgust was most often confused with anger.

\section{Relations between Fear Recognition and Perspective-Taking}

Statistical analyses for associations between fear recognition and perspective-taking were performed using R (RDCT, 2015). Due to the small sample size and, therefore, uncertain underlying distribution of the data, the data distribution was first checked for satisfaction of the normality assumption using the Shapiro-Wilk Test (Oztuna et al., 2006) and found to be violated. Given the small sample size and nonnormal distribution, a bootstrapping nonparametric approach (Efron and Tibshirani, 1993) was used to test the model (i.e., accuracy for fearful stimuli $=\beta 0+$ $\beta 1$ (perspective-taking scale on empathy total) $+\square$, where $\beta 0$ and $\beta 1$ are unknown model parameters and $\square$ is the measurement error). We estimated unknown parameters and calculated the $95 \%$ confidence interval of $\beta 1$ based on 1,000 bootstrapping procedure. Results show that the $95 \%$ confidence interval $(0.0182,6.665)$ does not contain zero, indicating a positive relationship between perspective-taking and accuracy in fear 
recognition. A Spearman correlation $(R=0.422 ; p=$ 0.036 ), conducted as a secondary analysis, further supports the finding. Perspective-taking did not correlate with any emotions (all $p$-values $>0.165$ ) except with fear. See Table 3 and 4 for complete Bootstrapping and Spearman correlation results.

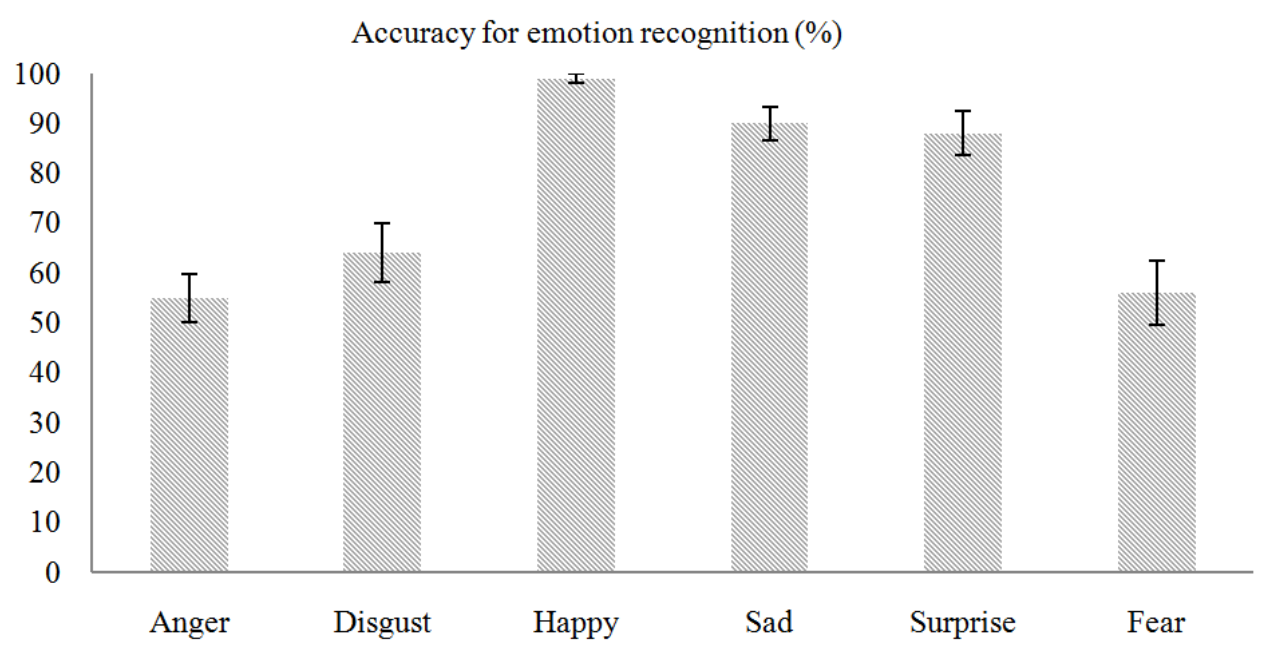

Fig. 1. Descriptive statistics for emotion recognition reported as percentage of accurately identified emotions for each emotion type

Table 2. Misattribution matrix from emotion recognition task

\begin{tabular}{|c|c|c|c|c|c|c|}
\hline \multirow{2}{*}{$\begin{array}{l}\text { Chosen } \\
\text { Emotion }\end{array}$} & \multicolumn{6}{|c|}{ Presented emotion } \\
\hline & Anger & Disgust & $\mathrm{Sad}$ & Fear & Surprise & Happy \\
\hline Anger & 55 & 34 & 1 & 1 & 1 & 1 \\
\hline Disgust & 17 & 64 & 3 & 32 & 0 & 0 \\
\hline $\mathrm{Sad}$ & 15 & 2 & 90 & 5 & 0 & 0 \\
\hline Fear & 11 & 0 & 1 & 56 & 11 & 0 \\
\hline Surprise & 1 & 0 & 5 & 4 & 88 & 0 \\
\hline Нарру & 0 & 0 & 0 & 2 & 0 & 99 \\
\hline
\end{tabular}

Table 3. Summary of results from bootstrapping approach

\begin{tabular}{llllll}
\hline & Mean & Median & SD & Boot CI $2.5 \%{ }^{\mathrm{a}}$ & ${\text { Boot CI } 97.5 \%{ }^{\mathrm{a}}}^{\mathrm{a}}$ \\
\hline Anger & -0.520 & -0.678 & 1.178 & -2.275 & 2.322 \\
Disgust & 1.115 & 1.182 & 1.860 & -3.093 & 4.564 \\
Fear & 2.861 & 2.705 & 1.7190 & 0.0182 & $6.665^{*}$ \\
Happy & $2.572 \mathrm{e}-01$ & $1.996 \mathrm{e}-01$ & $2.824 \mathrm{e}-01$ & $-1.159 \mathrm{e}-15$ & $9.529 \mathrm{e}-01$ \\
Sad & -0.546 & -0.602 & 0.968 & -2.337 & 1.531 \\
Surprise & -1.371 & -1.244 & 1.220 & -4.048 & 0.495 \\
Total & 0.277 & 0.197 & 0.489 & -0.479 & 1.465 \\
\hline
\end{tabular}

${ }^{\mathrm{a}}$ Boot CI 2.5\% and Boot CI 97.5\% represent the lower and upper bounds of 95\% confidence interval based on 1,000 bootstrapping procedure; *Indicates significant correlation where $95 \%$ CI contains zero

Table 4. Summary of results from Spearman correlation test

\begin{tabular}{lll}
\hline & Correlation Rho & $p$-value \\
\hline Anger & -0.087 & 0.362 \\
Disgust & 0.120 & 0.313 \\
Fear & 0.422 & $0.036^{*}$ \\
Happy & 0.237 & 0.164 \\
Sad & -0.222 & 0.181 \\
Surprise & -0.188 & 0.221 \\
Total & 0.029 & 0.452 \\
\hline
\end{tabular}

* indicates significant correlation 


\section{Discussion}

In a non-clinical adult sample, the ability to accurately detect fear in the faces of others was found to be positively correlated with perspective-taking abilities. This association is unique to recognition of fear, as perspective-taking was not significantly associated with recognition of the other basic emotions. Prior research has suggested a unique role for impaired fear recognition, more so than broad emotion recognition deficits, in multiple forms of psychopathology (e.g., Montagne et al., 2005; Weiss et al., 2006). These results extend these findings within a non-clinical sample and provide preliminary support for a possible mechanistic relationship between impaired fear recognition and impaired perspective-taking.

Results of this study suggest that perspective-taking is linked to fear recognition difficulties. Fear recognition ability, we assert, is a fairly stable individual difference that may predict perspective-taking as well as social functioning among non-psychiatric adults, as well as be predictive of psychopathology. What is unique about fear in terms of emotion recognition and perspectivetaking? When considering the possibility of emotionspecific impairments, it is important to examine the potential role of task difficulty. We found that emotion recognition accuracy in non-clinical adult males was highest for happiness, followed by sadness and surprise. Accuracy was lowest for anger and fear. These results are consistent with prior cross-cultural studies showing that recognition scores are highest for happiness and lowest for fear (Biehl et al., 1997). In addition, fear was most often misrecognized as disgust, followed by sadness, consistent with other findings (Kohler et al., 2014). Accuracy rates for fear expression are relatively low for non-clinical and clinical populations alike. Fear, however, is not more difficult to discriminate from neutral expression than are the other emotions (Adolphs, 2002) and, in the present study, we observed similarly low accuracy for other emotions, including anger and disgust.

Fear may be considered a valuable social signal, contributing to its association with perspective-taking. For example, fearful expression, in addition to sad facial emotion, is thought to serve as a social cue that conditions a person to avoid engaging in antisocial behaviors that elicit such expression (Marsh and Blair, 2008). Impairment in fear recognition could prevent a person (child or adult) from learning valuable social lessons, including appreciating other's perspectives. Impaired perspective-taking therefore could result from the impairment in recognizing facial expressions that are important in social interactions. Results from this study support this premise, given no other emotions showed a significant association with self-reported perspective- taking. Additionally, research has shown that similar past experiences, as well as the current mood, are important in perspective-taking abilities (Gerace et al., 2015). Negative emotions, such as fear, might be harder to elicit for some individuals which might compromise their ability to take other person's point of view.

Given the relationship between perspective-taking and fear recognition, we suggest that diminished perspective-taking may be an intervening process (or mechanism), through which impaired fear recognition ability contributes to social problems. It is also possible, however, that another factor (or factors), not considered in this study, explains the relationship found between fear recognition and perspective-taking. For example, motivation could be the mechanism contributing to the relationship, as individuals with deficits in recognition of fearful expressions could be less motivated to attend to and incorporate social (e.g., facial) cues which might results in lower perspective-taking in terms of lower motivation to show empathy. While this study shows the relationship between the processes, it does not exclude a mechanism not explored in this study.

Results should be evaluated in light of study limitations. First, the sample is small and homogeneous, comprised of twenty adult males. It is important to investigate the generalizability of the results to a broader population (i.e., females, children). Second, perspectivetaking in this study was measured with a single, selfreport trait measure. This limits the interpretation of the findings to a trait measure of empathy, or a consistent pattern of participant's behaviors and abilities, as opposed to their current state of empathy which might differ based on the situation. Future studies would benefit from exploring different aspects of perspectivetaking through a more comprehensive account of perspective-taking abilities. Although we showed an association between impairment in facial emotion recognition of fear and deficits in perspective-taking, future research needs to temporally establish the mechanism by which impairments occur and how this knowledge can be used to help individuals who show deficits in recognition of fearful facial expressions.

\section{Conclusion}

Impairments in perspective-taking and fear recognition have long been studied in numerous forms of psychopathology. This study shows a positive relationship between the two concepts. These results suggest that impaired perspective-taking may be mechanistically or developmentally linked to impaired ability to recognize facial emotion of fear. These findings represent an initial step toward establishing a potential mechanism for perspective-taking difficulties by showing a clear association between perspective- 
taking ability and deficits in fear recognition in a nonclinical sample. Given that facial recognition emerges before perspective-taking skills, it is likely that emotion recognition deficits lead to the difficulty with perspective-taking and not the other way around.

Transdiagnostic models allow for identification of fundamental processes underlying psychopathology. Such processes are believed to operate dimensionally, not limited to a specific setting or disorder and as such be present in non-clinical individuals without psychopathology as well. However, the mechanism(s) through which a risk process comes to manifest as problematic are not well understood (NolenHoeksema and Watkins, 2011). Our study raises several questions related to the non-clinical subject's ability to recognize fear expression from dynamic expressions of emotion, given the relative low accuracy of identifying fear compared to other emotions. These results have potential implications for understanding widespread processes that can affect non-clinical functioning as well as assessment and treatment approaches of disordered behavior. A richer understanding of the proximal effects of facial emotion recognition differences, specifically with respect to fear in others, in typical adults may inform clinical research, as it can help to identify possible modifiable mechanisms which can be targeted therapeutically.

\section{Acknowledgement}

This work was supported by a grant from the National Institutes of Mental Health (21MH100268: PI, White). We gratefully acknowledge the NIMH for its support.

\section{Author's Contributions}

Andrea Trubanova: Collected data, performed data analysis and drafted the manuscript.

Susan White: Conceived the study, designed the study, contributed to writing of the draft and revision of the manuscript.

Inyoung Kim: Performed statistical analysis, contributed to planning and design of the study, contributed to writing of the draft of the manuscript.

Marika C. Coffman, Martha Ann Bell, J. Anthony Richey, Stephen Laconte and Denis Gracanin: All contributed to planning and design of the study and writing of the draft of the manuscript.

\section{Ethics}

This article is original and contains unpublished material. The corresponding author confirms that all of the other authors have read and approved the manuscript and no ethical issues involved.

\section{References}

Adolphs, R., 2002. Recognizing emotion from facial expressions: Psychological and neurological mechanisms. Behav. Cognitive Neurosci. Rev., 1: 21-62. DOI: $10.1177 / 1534582302001001003$

Anastassiou-Hadjicharalambous, X. and D. Warden, 2008. Cognitive and affective perspective-taking in conduct-disordered children high and low on callous-unemotional traits. Child Adolescent Psychiatry Mental Health, 2: 16-16. DOI: $10.1186 / 1753-2000-2-16$

Aspan, N., C. Bozsik, J. Gardaros, P. Nagy and J. Inantsy-Pap et al., 2014. Emotion recognition pattern in adolescent boys with attentiondeficit/hyperactivity disorder. BioMed Res. Int., 2014: 761340-761347. DOI: 10.1155/2014/761340

Baron-Cohen, S., H. Tager-Flusberg and D.J. Cohen, 2000. Understanding other Minds: Perspectives from Developmental Cognitive Neuroscience. Oxford University Press, Oxford, ISBN-10: 0198524455, pp: 550.

Biehl, M., D. Matsumoto, P. Ekman, V. Hearn and K. Heider et al., 1997. Matsumoto and Ekman's Japanese and Caucasian Facial Expressions of Emotion (JACFEE): Reliability data and crossnational differences. J. Nonverbal Behav., 21: 3-21. DOI: $10.1023 / \mathrm{A}: 1024902500935$

Blair, R.J.R., E. Colledge, L. Murray and D.G.V. Mitchell, 2001. A selective impairment in the processing of sad and fearful expressions in children with psychopathic tendencies. J. Abnormal Child Psychol., 29: 491-498. DOI: $10.1023 / \mathrm{A}: 1012225108281$

Borkovec, T.D., J.L. Abel and H. Newman, 1995. Effects of psychotherapy on comorbid conditions in generalized anxiety disorder. J. Consult. Clin. Psychol., 63: 479-483. DOI: 10.1037/0022-006X.63.3.479

Coffman, M.C., L.C. Anderson, A.J. Naples and J.C. McPartland, 2015. Sex differences in social perception in children with ASD. J. Autism Dev. Disorders, 45: 589-599. DOI: $10.1007 / \mathrm{s} 10803-013-2006-5$

Corden, B., R. Chilvers and D. Skuse, 2008. Avoidance of emotionally arousing stimuli predicts socialperceptual impairment in Asperger's syndrome. Neuropsychologia, 46: 137-147. DOI: 10.1016/j.neuropsychologia.2007.08.005

Dadds, M.R., Y.E. Masry, S. Wimalaweera and A.J. Guastella, 2008. Reduced eye gaze explains "fear blindness" in childhood psychopathic traits. J. Am. Acad. Child Adolescent Psychiatry, 47: 455-463. DOI: $10.1097 / \mathrm{CHI} .0 \mathrm{~b} 013 \mathrm{e} 31816407 \mathrm{fl}$ 
Dalili, M.N., I.S. Penton-Voak, C.J. Harmer and M.R. Munafo, 2015. Meta-analysis of emotion recognition deficits in major depressive disorder. Psychol. Med., 45: 1135-1144. DOI: $10.1017 / \mathrm{S} 0033291714002591$

Davis, M.H., 1980. A multidimensional approach to individual differences in empathy. JSAS Catal. Selected Documents Psychol., 10: 85-85.

Davis, M.H., 1983. Measuring individual differences in empathy: Evidence for a multidimensional approach. J. Personality Soc. Psychol., 44: 113-126. DOI: $10.1037 / 0022-3514.44 .1 .113$

Demirel, H., D. Yesilbas, I. Ozver, E. Yuksek and F. Sahin et al., 2014. Psychopathy and facial emotion recognition ability in patients with bipolar affective disorder with or without delinquent behaviors. Comprehensive Psychiatry, 551: 542-546. DOI: 10.1016/j.comppsych.2013.11.022

Donno, R., G. Parker, J. Gilmour and D.H. Skuse, 2010. Social communication deficits in disruptive primary-school children. Brit. J. Psychiatry, 196: 282-289. DOI: 10.1192/bjp.bp.108.061341

Dziobek, I., K. Rogers, S. Fleck, M. Bahnemann and H. Heekeren et al., 2008. Dissociation of cognitive and emotional empathy in adults with asperger syndrome using the Multifaceted Empathy Test (MET). J. Autism Dev. Disorders, 38: 464-473. DOI: $10.1007 / \mathrm{s} 10803-007-0486-\mathrm{x}$

Efron, B. and R. Tibshirani, 1993. An Introduction to the Bootstrap. 1st Edn., Chapman and Hall, London, ISBN-10: 0412042312, pp: 456.

Fairchild, G., S.H.M. Van Goozen, A.J. Calder, S.J. Stollery and I.M. Goodyer, 2009. Deficits in facial expression recognition in male adolescents with early-onset or adolescence-onset conduct disorder. J. Child Psychol. Psychiatry, 50: 627-636. DOI: $10.1111 / \mathrm{j} .1469-7610.2008 .02020 . x$

Field, T.M., R.W. Woodson, R. Greenberg and C. Cohen, 1982. Discrimination and imitation of facial expression by neonates. Science, 218: 179-181. DOI: $10.1126 /$ science.7123230

Frick, A., W. Mohring and N.S. Newcombe, 2014. Picturing perspectives: Development of perspectivetaking abilities in 4- to 8-year-olds. Frontiers Psychol., 5: 386-386. DOI: 10.3389/fpsyg.2014.00386

Gerace, A., A. Day, S. Casey and P. Mohr, 2013. An exploratory investigation of the process of perspective taking in interpersonal situations. J. Relationships Res., 4: e6-e6. DOI: $10.1017 /$ jrr.2013.6

Gerace, A., A. Day, S. Casey and P. Mohr, 2015. Perspective taking and empathy: Does having similar past experience to another person make it easier to take their perspective? J. Relationships Res., 6: e10-e10. DOI: 10.1017/jrr.2015.6
Gibson, C.M., D.L. Penn, K.L. Smedley, J. Laserman and T. Elliott et al., 2014. A pilot six-week randomized controlled trial of oxytocin on social cognition and social skills in schizophrenia. Schizophrenia Res., 156: 261-265. DOI: 10.1016/J.SCHRES.2014.04.009

Gross, J.J. and H. Jazaieri, 2014. Emotion, emotion regulation and psychopathology: An affective science perspective. Clin. Psychol. Sci., 2: 387-401. DOI: $10.1177 / 2167702614536164$

Humphreys, K., N. Minshew, G.L. Leonard and M. Behrmann, 2007. A fine-grained analysis of facial expression processing in high-functioning adults with autism. Neuropsychologia, 45: 685-695. DOI: 10.1016/j.neuropsychologia.2006.08.003

Izard, C., S. Fine, D. Schultz, A. Mostow and B. Ackerman et al., 2001. Emotion knowledge as a predictor of social behavior and academic competence in children at risk. Psychol. Sci., 12: 18-23. DOI: 10.1111/1467-9280.00304

Kanade, T., J.F. Cohn and Y. Tian, 2000. Comprehensive database for facial expression analysis. Proceedings of the 4th IEEE International Conference on Automatic Face and Gesture Recognition, Mar. 28-30, IEEE Xplore Press, Grenoble, pp: 46-53. DOI: $10.1109 /$ AFGR.2000.840611

Kohler, C.G., T.H. Turner, R.E. Gur and R.C. Gur, 2014. Recognition of facial emotions in neuropsychiatric disorders. CNS Spectrums, 9: 267-274. PMID: 15048051

Lucey, P., J.F. Cohn, T. Kanade, J. Saragih and Z. Ambadar et al., 2010. The Extended Cohn-Kanade Dataset $(\mathrm{CK}+)$ : A complete dataset for action unit and emotion-specified expression. Proceedings of the 3rd IEEE Workshop on CVPR for Human Communicative Behavior Analysis, Jun. 13-18, IEEE Xplore Press, San Francisco, CA., pp: 94-101. DOI: $10.1109 /$ CVPRW.2010.5543262

Marsh, A.A. and R.J.R. Blair, 2008. Deficits in facial affect recognition among antisocial populations: A meta-analysis. Neurosci. Biobehav. Rev., 32: 454-465. DOI: 10.1016/j.neubiorev.2007.08.003

Marsh, D.T., F.C. Serafica and C. Barenboim, 1981. Interrelationships among perspective taking, interpersonal problem solving and interpersonal functioning. J. Genetic Psychol.: Res. Theory Human Dev., 138: 37-48. DOI: $10.1080 / 00221325.1981 .10532840$

Montagne, B., J. van Honk, R.P.C. Kessels, E. Frigerio and M. Burt et al., 2005. Reduced efficiency in recognising fear in subjects scoring high on psychopathic personality characteristics. Personality Individual Differences, 38: 5-11. DOI: 10.1016/j.paid.2004.02.008 
Nantel-Vivier, A., R.O. Pihl, S.N. Young, S. Parent and S.A. Belanger et al., 2011. Serotonergic contribution to boys' behavioral regulation. PLoS One. DOI: 10.1371/journal.pone.0020304

Nolen-Hoeksema, S. and E.R. Watkins, 2011. A heuristic for developing transdiagnostic models of psychopathology: Explaining multifinality and divergent trajectories. Perspective Psychol. Sci., 6: 589-609. DOI: 10.1177/1745691611419672

Oztuna, D., A.H. Elhan and E. Tuccar, 2006. Investigation of four different normality tests in terms of type 1 error rate and power under different distributions. Turkish J. Med. Sci., 36: 171-6.

RDCT, 2015. R: A language and environment for statistical computing. Vienna, Austria: R Foundation for Statistical computing.

Seidel, E.M., D.M. Pfabigan, K. Kecheis, A.M. Wucherer and T. Jahn et al., 2013. Empathic competencies in violent offenders. Psychiatry Res., 210: 1168-1175.

DOI: $10.1016 /$ j.psychres.2013.08.027

Silverman, W.K. and A.M. Albano, 1996. Anxiety Disorders Interview Schedule for Children. 1st Edn., Psychological Corporation, San Antonio, TX.

Skuse, D., 2003. Fear recognition and the neural basis of social cognition. Child Adolescent Mental Health, 8: 50-60. DOI: 10.1111/1475-3588.00047
Sodian, B., C. Thoermer and U. Metz, 2007. Now I see it but you don't: 14-month-olds can represent another person's visual perspective. Dev. Sci., 10: 199-204. DOI: $10.1111 /$ j.1467-7687.2007.00580.x

Stevens, D., T. Charman and R.J.R. Blair, 2001. Recognition of emotion in facial expressions and vocal tones in children with psychopathic tendencies. J. Genetic Psychol., 162: 201-211.

DOI: 10.1080/00221320109597961

Uljarevic, M. and A. Hamilton, 2013. Recognition of emotions in autism: A formal meta-analysis. J. Autism Dev. Disorders, 43: 1517-1526. DOI: $10.1007 / \mathrm{s} 10803-012-1695-5$

Weiss, E.M., C.G. Kohler, K.A. Nolan, P. Czobor and J. Volavka et al., 2006. The relationship between history of violent and criminal behavior and recognition of facial expression of emotions in men with schizophrenia and schizoaffective disorder. Aggressive Behav., 32: 187-194. DOI: $10.1002 / \mathrm{ab} .20120$

Wechsler, D., 2011. Wechsler Abbreviated Scale of Intelligence. 2nd Edn., The Psychological Corporation, San Antonio, TX. 